The authors demonstrate that marketing actions, such as pricing, can alter the actual efficacy of products to which they are applied. These placebo effects stem from activation of expectancies about the efficacy of the product, a process that appears not to be conscious. In three experiments, the authors show that consumers who pay a discounted price for a product (e.g., an energy drink thought to increase mental acuity) may derive less actual benefit from consuming this product (e.g., they are able to solve fewer puzzles) than consumers who purchase and consume the exact same product but pay its regular price. The studies consistently support the role of expectancies in mediating this placebo effect. The authors conclude with a discussion of theoretical, managerial, and

public policy implications of the findings.

\title{
Placebo Effects of Marketing Actions: Consumers May Get What They Pay For
}

\author{
Pro tali numismate tales merces. [One gets what one \\ pays for.] \\ —Gabriel Biel (Dictionary of Clichés, Rogers 1985)
}

Consumers' beliefs and expectations, shaped by experiences in their daily lives, often influence their judgments of products and services. For example, consumers often believe and, therefore, judge lower-priced items to be of lower quality (see, e.g., Gerstner 1985; Huber and McCann 1982; Rao and Monroe 1989). Consumers' beliefs and expectations can also affect their subjective experiences. For example, a drink may taste better if it has a favorite brand's label than if it is unlabeled (Allison and Uhl 1964; McClure et al. 2004). Similarly, meat that is labeled $75 \%$ fat free tastes better than the same meat that is labeled as containing 25\% fat (Levin and Gaeth 1988). The question that we address in this research is whether beliefs and expectations that marketing actions evoke can affect more than judgments and subjective consumption experiences. Specif-

*Baba Shiv is Associate Professor of Marketing, Graduate School of Business, Stanford University (e-mail: shiv_baba@gsb.stanford.edu). Ziv Carmon is Associate Professor of Marketing, INSEAD, Asia Campus (e-mail: ziv.carmon@insead.edu). Dan Ariely is Luis Alvarez Renta Professor of Management Science, Massachusetts Institute of Technology (e-mail: ariely@mit.edu). The authors thank Himanshu Mishra, Chris Taylor (parts of this research formed the basis of his honors thesis), and Monica Wadhwa for their help in administering the experiments and coding participants' responses. They also thank Eric Johnson, participants of the Distinguished Scholars Retreat at the University of Alberta, and the three anonymous $J M R$ reviewers for their comments and suggestions. We dedicate this article to the late Dick Wittink for his exceptional guidance and encouragement. May he rest in peace.

Editor's note: In contrast to other lead articles that were invited by former editor Dick Wittink, this article is based on a regular submission. ically, can they also influence the actual efficacy of the marketed product? For example, can consuming an energy drink that is purchased at a discount lead not only to judgments of lower quality or to a less favorable consumption experience but also to diminished performance in, for example, a cardiovascular workout or a puzzle-solving task?

We began to explore these questions in a preliminary study in which 38 members of a fitness center who exercised regularly (at least three times a week) consumed Twinlab Ultra Fuel before and during a workout session. Before consuming the energy drink, participants were shown the list of its ingredients and were told that the drink was from the most recently manufactured batch. One group of participants was told that we purchased the drink at the regular price of $\$ 2.89$; another group was told that the regular price of the drink was $\$ 2.89$ but that we had purchased it at a discounted price of $\$ .89$ because we bought it in bulk as an institutional purchase. After exercising, participants rated the intensity of their workout on a scale that ranged from -3 ("not at all intense") to +3 ("very intense") and how fatigued they felt on a scale that ranged from 1 ("not at all") to 7 ("very"). The results show that participants in the reduced-price condition rated their workout intensity as lower $(\mathrm{M}=-.4)$ than did those in the regular-price condition $(\mathrm{M}=.6 ; \mathrm{F}(1,36)=7.5, p<.01)$, and participants in the reduced-price condition indicated that they were more fatigued $(\mathrm{M}=4.5)$ than did those in the regular-price condition $(\mathrm{M}=3.7 ; \mathrm{F}(1,36)=3.5, p<.10)$. Finally, when asked during debriefing if the price of the drink affected the workout, not a single participant answered affirmatively.

The findings of our preliminary study share a kinship with the well-known placebo phenomenon in the medical 
domain (see Stewart-Williams and Podd 2004). Specifically, patients' beliefs and expectations about the treatment they are receiving (e.g., an antidepression medication) can yield real changes to their health, even if the treatment is actually inert and has no inherent power to produce health effects (e.g., an inert sugar pill that looks like the antidepression medication). A large body of research has examined effects of aspects that are inherent to a placebo, such as beliefs about the efficacy of a drug for which the placebo is a substitute (e.g., Kirsch 1999) or about the form in which the placebo is received (e.g., Kaptchuk et al. 2000). The results of our preliminary study suggest that features that are not inherent to a product, such as its price, can also trigger a placebo effect.

The preliminary study addresses the questions we raised in the first paragraph, and its results suggest that price discounts can lead to a behavioral effect-we refer to this as a placebo effect of marketing action in this research-and that this effect may occur beyond awareness. However, several criticisms can be leveled against this study. First, our dependent measure in this study is our participants' perceptions of their behavior (e.g., perceived workout intensity) rather than the behavior itself. Second, our study does not include a no-treatment control group. Therefore, we cannot distinguish between a desirable placebo effect (i.e., the regular-price fitness drink boosted the efficacy of the drink) and an undesirable placebo effect (i.e., the sale price detracted from the efficacy of the energy drink). ${ }^{1}$ Third, participants did not actually pay for the drink but were merely informed of the prices. Finally, and most important, this study gives no indication of what underlies the effect. We address all these criticisms in our subsequent studies and also explore whether the effect is nonconscious, as the preliminary study suggests.

In the next section, we draw on research on the placebo effect and on the price-quality association to predict how beliefs and expectations that arise from marketing actions, such as price promotions, may produce effects on behavior. Following this, we present three experiments that document undesirable placebo effects that result from price discounts. In the third experiment, we also document a desirable

${ }^{1}$ Although some researchers (e.g., Hahn 1997) distinguish between placebo and nocebo effects, we use the term placebo for both, in line with the common view that the desirability of the effect should not influence its labeling (Stewart-Williams and Podd 2004). placebo effect that is ignited by advertising claims. In all three experiments, we find support for the role of expectancies as underlying this effect, and we rule out alternative accounts.

\section{THEORETICAL BACKGROUND}

Voluminous research on placebo effects has shown that successfully conveying the false belief that patients received a particular treatment can bestow some of the benefits of the genuine treatment (for a review, see Stewart-Williams and Podd 2004). Credible placebos can help relieve and sometimes even cure physical and mental illness, such as pain (e.g., Montgomery and Kirsch 1996), cardiovascular disease (e.g., Bienenfeld, Frishman, and Glasser 1996), and depression (Kirsch and Sapirstein 1999). Placebo effects have also been detected with functional magnetic resonance imaging (fMRI; Wager et al. 2004).

Two notions are believed to account for placebo effects: expectancy theory and classical conditioning. According to expectancy theory, placebo effects arise because beliefs about a substance/procedure serving as a placebo activate expectations that a particular effect will occur, which then affect the subsequent effectiveness of the substance/ procedure. The classical conditioning view considers consuming substances with known therapeutic effects to be conditioning trials. The active substances giving rise to these effects serve as unconditioned stimuli, and the vehicles by which they are delivered (e.g., pills, capsules, drinks) serve as the conditioned stimuli. Pairing the unconditioned stimuli and the conditioned stimuli over time endows the vehicles with a capacity to evoke therapeutic effects in the form of conditioned responses. These two views have been contrasted and debated, but an emerging view is that expectancies mediate all placebo effects, and conditioning is one means by which people initially form and then activate expectancies (Kirsch 2004; Rescorla 1988). The growing acceptance of expectations as the basic mechanism for placebo effects has led to an increased interest in how beliefs lead to placebo effects and the role of expectancies in mediating this effect.

\section{The Mediating Role of Expectancies}

Figure 1 highlights the process associated with placebo effects, a framework that we derive from work in the medical domain (Kirsch 1999; Kirsch and Lynn 1999; Kirsch

Figure 1

FRAMEWORK FOR PLACEBO EFFECTS

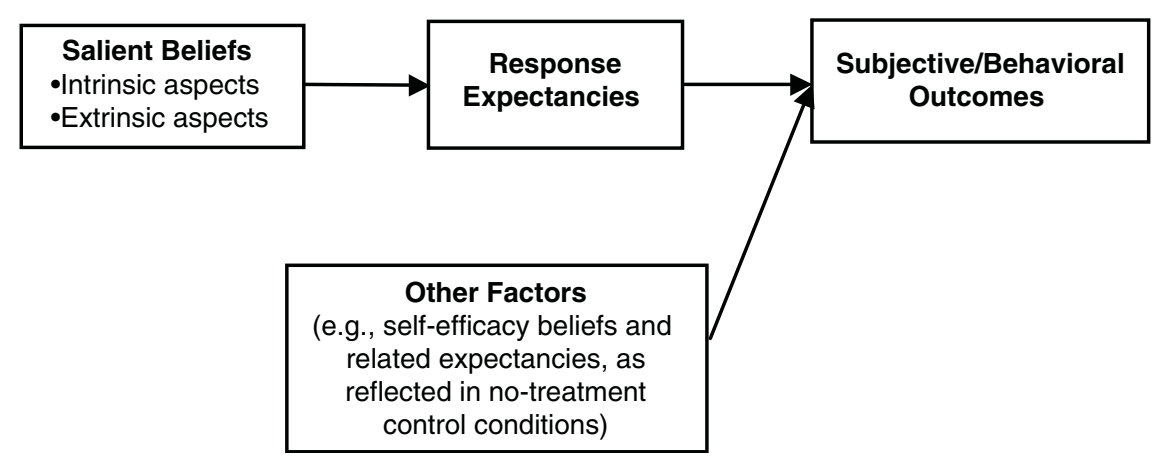


and Sapirstein 1999; Stewart-Williams and Podd 2004). The figure presents factors that are expected to influence placebo effects of marketing actions. Briefly, when a person receives what is purportedly an active substance or treatment, his or her salient beliefs about the substance or treatment activate response expectancies, or anticipations of subjective and/or behavioral consequences of using the substance or being treated. These response expectancies along with contextual factors that are unrelated to the substance or treatment then lead to the subjective and behavioral outcomes, or placebo effects.

Several aspects of this process warrant elucidation. First, critical to the placebo effect are specific beliefs that are salient when a person receives the purportedly active substance or treatment. These beliefs, for example, could be related to intrinsic aspects of the active substance or treatment, such as its potential therapeutic effects or its deleterious side effects, yielding a desirable placebo effect in the former case and an undesirable placebo effect in the latter case (see Hahn 1997). Similarly, extrinsic aspects can shape salient beliefs about the substance or treatment and lead to stronger (weaker) placebo effects. An example of this is whether a person receives a medication through injections or capsules (Kaptchuk et al. 2000).

Second, the magnitude of the subjective and/or behavioral consequences depends on the strength of the activated response expectancies, which in turn can be influenced by a variety of factors. For example, encouraging people to elaborate on their expectations may enhance the magnitude of the placebo effect (Fillmore and Vogel-Sprott 1992). Similarly, the magnitude of the placebo effect may be influenced by the strength of a person's salient beliefs about the active substance or treatment. In turn, for example, this can be enhanced by greater familiarity through prior usage (Kirsch 1985). The strength of these beliefs and, therefore, the magnitude of the placebo effect may also be diminished by instructions that cast doubts about these beliefs. For example, alerting people that they are participating in a doubleblind study and that the substance they are receiving may be inert gives rise to diminished placebo effects (Kirsch and Weixel 1988).

Third, the subjective and behavioral outcomes can be shaped by self-efficacy beliefs (Kirsch 1985), which, together with other extraneous factors, are reflected in outcomes of no-treatment control conditions that are sometimes included in placebo studies. Finally, the process by which expectancies are elicited to lead to the placebo effect can be either conscious or nonconscious. Consistent with one aim of our research, Stewart-Williams and Podd (2004) call for research on placebo effects that will identify situations in which the mediating role of expectancies occurs nonconsciously. Next, we examine implications of the framework for placebo effects that may arise from marketing actions, such as price discounts.

\section{Marketing Actions and the Placebo Effect}

If marketing actions, such as price discounts, lead to a placebo effect, as our preliminary study suggests, what might be the nature of beliefs that trigger response expectancies that, in turn, lead to the placebo effect? Furthermore, how will contextual factors influence the strength of the expectancies and, thus, the magnitude of the effect? To answer these questions, consider the context of the pre- liminary study. Recall that participants received an energy drink (Twinlab Ultra Fuel), were shown a list of its ingredients, and were informed that we purchased the drink at either its regular price or a discounted price. The stimulus materials could have made several beliefs salient. For example, intrinsic aspects related to the ingredients could have activated beliefs about their effects. In addition, the brand name (an extrinsic cue) could have activated beliefs about the product's superior quality (Rao and Monroe 1989). Furthermore, given that consumers often believe that price levels tend to reflect quality (e.g., Huber and McCann 1982; Rao and Monroe 1988, 1989), the price discount (another extrinsic cue) may have triggered beliefs that the product's quality was inferior. According to the framework we presented previously, all these beliefs could have been salient, triggering various types of response expectancies. These response expectancies along with other factors, such as non-product-related beliefs (e.g., self-efficacy beliefs, such as how good a person is at fitness workouts) and participants' abilities, could have affected respondents' performance in their fitness workout. However, because price was the only manipulated factor in the preliminary experiment and because participants were randomly assigned to the two levels of this factor (thereby controlling for other factors, such as beliefs about the ingredients, the brand name, or self-efficacy), the difference we observed in participants' performance is likely to have been due to the salient beliefs about price, that is, a placebo effect of price discounts. Conversely, participants' performance in a no-treatment control condition would have reflected effects of the other factors, such as non-product-related beliefs, participants' abilities, and so forth.

Next, we consider the implications of other aspects of the framework in relation to our preliminary study, and we draw predictions for similar studies we present subsequently. The magnitude of the placebo effect could be affected by a host of factors. First, encouraging participants to elaborate on their expectations could increase the strength of those expectations and, thus, the magnitude of the observed placebo effect. We test this prediction in our first study. Second, beliefs about the brand name and/or the ingredients should be stronger with greater (rather than lower) frequency of prior usage. Thus, in addition to the observed price effect, we expect that frequency of prior usage influences the behavioral outcome. We provide evidence relating to prior usage in our first two studies. Third, if we draw participants' attention to their price-quality beliefs, many may realize that the price-quality relationship is not applicable, which should weaken the impact of such beliefs and, thus, the magnitude of the placebo effect. We test this prediction in our second study. Finally, consider what might happen if we presented advertising claims that either strengthened or weakened participants' beliefs in the efficacy of the ingredients (an intrinsic cue). This manipulation should have independent effects on participants' performance, in addition to the observed placebo effect of price discounts. We examine this in the third study.

The discussion in the previous section also suggests that the process by which expectancies are elicited to lead to the placebo effect can be either conscious or nonconscious (see Kirsch 2004; Stewart-Williams and Podd 2004). Thus, the question is whether the placebo effects of price discounts are conscious. Rao and Monroe (1988) argue that the rela- 
tionship between price and perceived quality is a belief that is activated and used when people make rapid judgments about a product's quality. Adaval and Monroe (2002) suggest that price-quality beliefs are activated and affect judgments at a nonconscious level.

Building on these ideas, we conducted three experiments. In these experiments, we investigated whether price discounts lead to placebo effects by activating response expectations and whether the process by which these activated expectations lead to the placebo effect is in line with our previous predictions.

\section{EXPERIMENT 1}

The purpose of Experiment 1 was to (1) document evidence of a placebo effect caused by price discounts; (2) explore what underlies the placebo effect by examining whether the observed effects are mediated by expectancies, in line with findings in the medical domain; and (3) determine whether the impact of expectancies on the observed placebo effect occurs nonconsciously.

In this experiment, participants first consumed SoBe Adrenaline Rush (a drink that claims to help increase mental acuity on its package) and then solved a series of puzzles. Note that such drinks are familiar to the student population from which we derived our sample. Indeed, in response to measures we collected at the end of this experiment, $92 \%$ of the participants stated that they had heard of SoBe before, and $48 \%$ stated that they had consumed this drink before. To accomplish the first goal of this experiment, we collected a measure of performance, namely, the number of puzzles solved correctly. To accomplish the second goal, we adapted a procedure that Fillmore and VogelSprott (1992) used to vary the strength of activated response expectancies. Specifically, one group of participants elaborated on their expectancies by rating the expected efficacy of the drink before solving the puzzles, and a second group did not perform this rating task. If expectancies indeed mediate an observed placebo effect, strengthening response expectancies should amplify the basic effects. To accomplish the third objective, we used a straightforward dependent variable approach that we adapted from previous work on nonconscious processes (see, e.g., Fitzsimons, Chartrand, and Fitzsimons 2004). After participants completed the puzzle task, we asked them to rate how effective SoBe was at improving their puzzle-solving performance on a scale that ranged from 1 ("not at all effective") to 7 ("very effective"). If participants were (non)conscious of the impact of expectancies related to the efficacy of SoBe on their subsequent performance, this measure should (not) mediate the effects of the independent variables on the number of puzzles solved. Note that the underlying process has two components: (1) activation of expectancies and (2) subsequent impact of those expectancies on participants' performance in the puzzle task. Even if the first component occurs at a nonconscious level, the procedure we used to strengthen expectancies would make expectancies conscious (in conditions in which participants were asked to rate the efficacy of the drink). Thus, lack of mediation would suggest only that the second component occurs nonconsciously. We explore the nature of the first component in Experiment 2 because our approach in Experiment 1 does not enable us to learn about it.

\section{Design and Procedure}

We used a 2 (price: regular versus discounted) $\times 2$ (expectancy strength: high versus low) between-subjects design. We randomly assigned 125 participants to the four conditions. At the beginning of the session, participants were told that as part of the study, they would consume SoBe. As in the preliminary study, they were shown the packaging and the ingredients it contained and were told that the drink was from the most recently manufactured batch. To reinforce the sense that the energy drink would influence their performance, participants were then told that they would watch a video for about ten minutes, purportedly to allow the ingredients to have their effects. They were also told that after watching the video, they would solve a series of word-jumble puzzles (e.g., TUPPIL, the solution for which is PULPIT); their goal was to solve as many puzzles as possible in the allotted 30 minutes. Before the drink was distributed, participants were given a form that authorized us to charge their university billing account for the drink that they would consume. For some participants (regular-price conditions), the form stated that they would be charged $\$ 1.89$ and that this was the regular price of the drink in retail outlets. For other participants (reducedprice conditions), the form stated that the regular price of the drink at retail outlets was $\$ 1.89$ but that they would be charged $\$ .89$ because we purchased the drink at a discount as part of an institutional purchase.

Participants consumed the drink and then watched a video for about ten minutes. They then received a booklet that contained instructions on the cover page, followed by the puzzles. The instructions on the cover sheet stated that participants would have 30 minutes to solve 15 puzzles. After the cover page, some respondents (high-expectancystrength conditions) were shown a page with the following statements: "I feel that SoBe is "very bad' (1)/"very good" (7) at improving concentration," and "I feel that SoBe is 'very bad' (1)/“very good' (7) at improving mental performance." Respondents in the low-expectancy-strength conditions were not shown these statements. Subsequently, participants engaged in the puzzle task and then responded to a series of measures; finally, they were debriefed.

\section{Other Measures}

After solving the puzzles, participants indicated their gender; whether they were familiar with SoBe; whether they had consumed this drink before; and, in general, how good and how experienced they were at solving puzzles such as word jumbles. These measures served as covariates in the various analyses. At the end of the instrument, we asked participants to recall the price they paid for the drink (in this and in our other experiments, all participants in the treatment conditions recalled the price they had paid within a range of $+/-11 \%$ accuracy, and there were no differences in recall across the various treatment conditions). After the experiment, an independent coder determined the number of puzzles that each respondent solved correctly.

\section{Results}

Pilot study. We first conducted a pilot study to assess participants' performance in a no-treatment (control) condition. For the pilot study, we used 31 participants from the same population as that of the main experiment. The procedure 
closely followed the one we used in the main experiment, except that the participants were not told about the SoBe drink and did not consume it; they merely solved the puzzles and responded to a relevant subset of the measures. The average number of puzzles solved by the participants of the pilot study was 9.1 .

Main experiment. The average number of puzzles solved across the various conditions appears in Figure 2. An analysis of covariance (ANCOVA) on the number of puzzles solved revealed an interaction between price and expectancy strength $(\mathrm{F}(1,120)=5.6, p<.05)$ and a main effect of price $(\mathrm{F}(1,120)=34.7, p<.0001)$. The pattern of results in the low-expectancy-strength conditions was consistent with a placebo effect; the number of puzzles solved was lower in the reduced-price condition $(\mathrm{M}=7.7)$ than in the regular-price condition $(\mathrm{M}=9.5 ; \mathrm{F}(1,120)=5.9, p<$ $.05)$.

The results in the high-expectancy-strength conditions suggest that the observed placebo effect was indeed mediated by expectancies about the efficacy of the drink. As Figure 2 shows, when expectancy strength was high, the magnitude of the undesirable placebo effect in the lowexpectancy-strength conditions increased. Specifically, when the price was discounted, the number of puzzles solved decreased further at higher $(\mathrm{M}=5.8)$ rather than lower $(\mathrm{M}=7.7)$ levels of expectancy strength $(\mathrm{F}(1,120)=$ $7.7, p<.01)$.

A Sobel test (e.g., MacKinnon et al. 2002) within the high-expectancy-strength conditions further supports mediation by expectancies. Recall that we operationalized expectancy strength by asking respondents in the highexpectancy-strength conditions to rate how good SoBe was at improving concentration and mental performance before they solved the puzzles (we did not ask respondents in the low-expectancy-strength conditions this question). In line with our conceptualization, these ratings were higher in the regular-price condition $(\mathrm{M}=4.3)$ than in the reduced-price condition $(\mathrm{M}=3.5 ; \mathrm{F}(1,64)=11.1, p<.01)$. The Sobel test revealed that these ratings also mediated the effects of the independent variable, price, on the number of puzzles

\section{Figure 2}

NUMBER OF PUZZLES SOLVED: EXPERIMENT 1

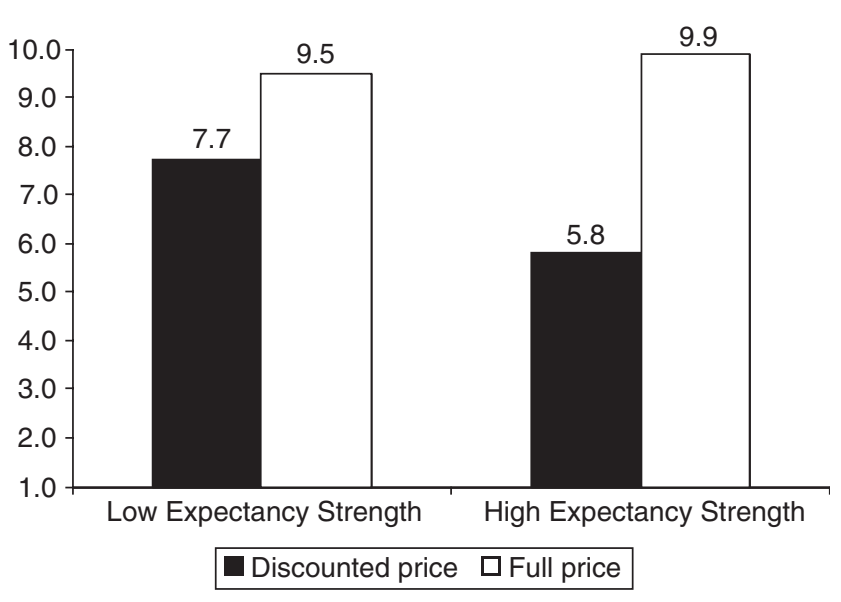

Notes: The number of puzzles solved in the control condition $=9.1$. solved within the high-expectancy-strength conditions $(\mathrm{z}=$ $3.0, p<.01)$.

The only covariate that was relevant in the ANCOVA was prior consumption of $\mathrm{SoBe}$, a variable that did not interact with any of the other independent factors. To explore the effects of prior usage further, we performed an analysis of variance with prior usage, a categorical variable, as a third independent factor. Again, the only effect we observed for prior usage was a main effect; on average, participants who had consumed SoBe before solved more puzzles $(\mathrm{M}=8.7)$ than did those who had not $(\mathrm{M}=7.7 ; \mathrm{F}(1,117)=4.2, p<$ $.05)$, as we predicted in our framework.

Was the underlying process conscious? To answer this question, we examined participants' responses to a measure that we collected after they solved the puzzles. Recall that after participants solved the puzzles, they were asked how effective they believed SoBe was at improving their puzzlesolving performance $(1=$ "not at all effective," 7 = "very effective"). Had participants been conscious that their performance was affected by consumption of the drink, this measure would have mediated the effects of the independent variables on the number of puzzles solved. A Sobel test did not support this possibility $(p=.64)$, suggesting that expectancies were not conscious when participants solved the puzzles. However, note that this null result is difficult to interpret because it could have occurred for reasons other than the process being nonconscious. We test the potential nonconscious nature of the underlying process more explicitly in the next experiment.

\section{Discussion}

The results of Experiment 1 support our basic prediction that price discounts can lead to an undesirable placebo effect. Offering a price discount on a product that claims to be beneficial for mental acuity negatively affected performance on a subsequent task, namely, the number of puzzles solved correctly. The performance of participants who consumed the discounted drink was worse than that of participants who consumed the regular-priced drink and of those who were in the no-treatment pilot study (and did not consume the drink). This detrimental effect was accentuated when we reinforced expectations about the efficacy of the product. Furthermore, on average, participants who had consumed the energy drink before solved more puzzles than did those who had not. However, this variable did not interact with any of the other independent variables. Finally, the results suggest that the underlying process leading to our observed placebo effect occurred nonconsciously.

A noteworthy finding in Experiment 1 was that we observed only an undesirable placebo effect in the reducedprice conditions. The results in the regular-price conditions were no different than the results in the no-treatment control condition (which we administered as a pretest). In the next experiment, we examine whether the findings of Experiment 1 replicate and shed more light on the cause of the observed placebo effect.

\section{EXPERIMENT 2}

One goal of Experiment 2 was to examine why we did not observe a desirable placebo effect of the regular-priced drink in Experiment 1. A second goal was to rule out two alternative accounts: (1) that participants paying the regular 
price might have worked harder on the puzzle task to reduce the greater dissonance they might have experienced as a result of the regular price they paid and (2) that compared with participants in the regular-price condition, those in the reduced-price conditions concentrated less on the puzzle task as a result of entertaining distracting thoughts about getting the drink at a lower price. A third goal of Experiment 2 was to examine whether drawing attention to pricequality beliefs would affect the observed placebo effect. This enables us to test several predictions. First, drawing participants' attention to price-efficacy beliefs is likely to help some of them realize that these beliefs may not be applicable to all contexts. In turn, this should weaken their response expectations and, thus, the magnitude of the placebo effect (see Kirsch and Weixel 1988). ${ }^{2}$ Second, the procedure enables us to shed more light on whether the underlying process is nonconscious. Research has consistently shown that if the activation of information in memory occurs nonconsciously, drawing attention to the priming source (in our case, the relationship between price and expected efficacy) reduces subsequent effects of this information (e.g., Strack et al. 1993). This attenuating effect is likely to occur when drawing attention to the priming source casts doubts on the relevance of the priming source. Conversely, if the activation of the information occurs consciously, drawing attention to the priming source enhances subsequent effects of the information. Third, if drawing participants' attention to the price-efficacy beliefs reduced the magnitude of the observed placebo effect, it would reduce the viability of the alternative cognitive dissonance account. According to the dissonance explanation, drawing attention to the price of the drink should increase dissonance in the regular-price condition, thus enhancing rather than attenuating the magnitude of the basic effect.

To accomplish the third goal of examining whether drawing attention to price-quality beliefs affects the placebo effect, we modified the procedure that we used in Experiment 1 to strengthen expectancies before the puzzle task. Recall that in Experiment 1, we manipulated the strength of expectancies by having one group of participants respond to the following statements before the puzzle task: "I feel that SoBe is 'very bad' (1)/“very good' (7) at improving concentration," and "I feel that SoBe is 'very bad' (1)/“very good' (7) at improving mental performance." In Experiment 2, one group of participants rated similar statements, except that their attention was also drawn to the price-efficacy link by the following words that we added to the beginning of the statements: "Given the price I was charged for SoBe,..."

To summarize, Experiment 2 used a 2 (price: regular versus discounted) $\times 2$ (price-efficacy salience: low versus high) between-subjects design and a control condition.

\footnotetext{
2This assumption was supported in a separate pretest. We used 33 participants from the same population as that of the main studies and asked them to perform a task similar to that in Experiment 1. Participants were presented with the energy drink, $\mathrm{SoBe}$, and informed that its regular price was $\$ 1.89$, but they would buy it from us at a discounted price of $\$ .89$. Following a filler task, participants rated the perceived efficacy of the drink (as in the high-expectancy-strength conditions of Experiment 1). However, for one group of participants, the price-efficacy link was made salient before they rated the drink (they were told, "Given the price of SoBe, please rate..."). Consistent with our assumption, the mean ratings were lower when the price-efficacy link was made salient $(M=3.8)$ than when it was not $(\mathrm{M}=4.9 ; \mathrm{F}(1,31)=7.6, p<.01)$.
}

Apart from modifying the task to incorporate the priceefficacy salience factor, adding measures to serve as covariates, and conducting Experiment 2 using computers, the procedure paralleled the one we used in Experiment 1 (our using a computerized rather than a paper-and-pencil task as in Experiment 1 may account for some differences in the performance levels across Experiments 1 and 2). A total of 193 undergraduate students participated in the study.

\section{Results}

The average number of puzzles solved across the various conditions appears in Figure 3. An ANCOVA on the number of puzzles solved revealed an interaction between price and price-efficacy salience $(\mathrm{F}(1,189)=5.1, p<.05)$ and a main effect of price $(\mathrm{F}(1,189)=3.1, p<.10)$. As in Experiment 1 , prior consumption of $\mathrm{SoBe}$ was a relevant covariate that did not interact with any other factor, and on average, participants who had consumed SoBe before solved more puzzles than did those who had not (see Kirsch 1985).

The results in the low price-efficacy salience conditions paralleled those in the low-expectancy-strength conditions of Experiment 1. As in Experiment 1 and consistent with a placebo effect of price discounts, the number of puzzles solved was lower in the reduced-price condition $(\mathrm{M}=6.8)$ than in the regular-price condition $(\mathrm{M}=8.3 ; \mathrm{F}(1,189)=$ $7.8, p<.01)$ and the control condition $(\mathrm{M}=8.0 ; \mathrm{F}(1,189)=$ $5.0, p<.05)$. In addition, the number of puzzles solved was not different between the regular-price and the control conditions $(\mathrm{F}<1)$. As in Experiment 1, we obtained only an undesirable placebo effect of discounted prices and no desirable placebo effect of the regular-priced drink. We discuss this finding subsequently.

The results in the high price-efficacy salience conditions suggest that drawing attention to price-efficacy beliefs weakens these beliefs, thus eliminating the placebo effect $(\mathrm{Ms}=8.2$ and 8.0, respectively, in the reduced- and regularprice conditions). In addition, that drawing attention to the

Figure 3

NUMBER OF PUZZLES SOLVED: EXPERIMENT 2

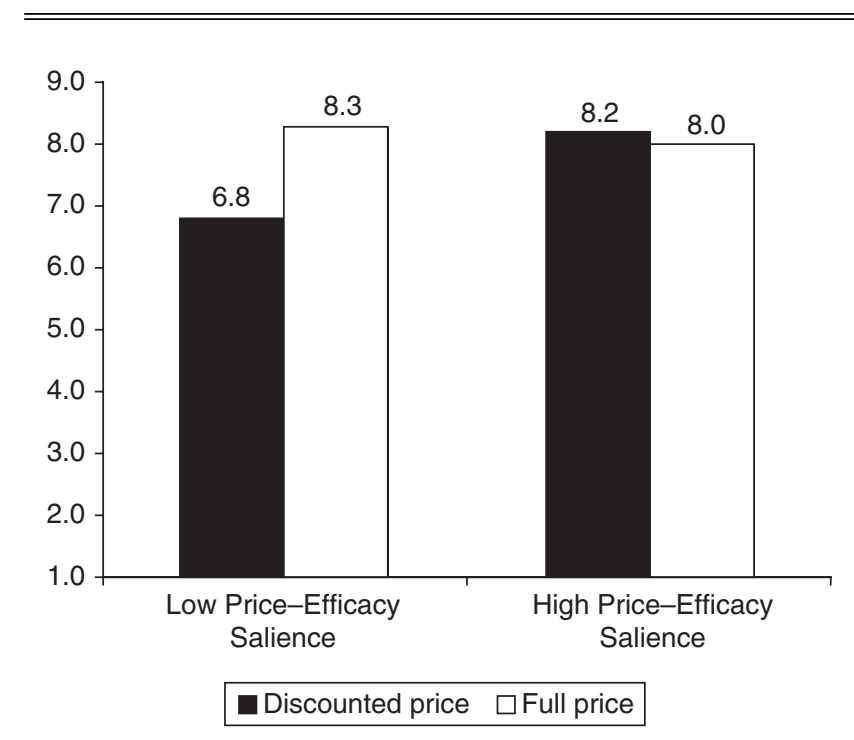

Notes: The number of puzzles solved in the control condition $=8.0$. 
price-efficacy link eliminated the placebo effect is consistent with the initial activation of expectancies being nonconscious (see Strack et al. 1993). In further reducing the viability of this alternative account, drawing attention to the price of the drink not only eliminated the basic effect but also resulted in means that were in the opposite direction of what the cognitive dissonance and the distraction explanations predict. Finally, note that both of these alternative accounts rely on participants paying for their drink in our experiments. However, although participants in the fitness study we presented in the introduction did not pay for the energy drink, we observed a placebo effect in that study.

We found further support for our conceptualization by examining the expectancy measures that we collected in the high price-efficacy salience conditions before the puzzlesolving task (to operationalize the price-efficacy salience factor). According to our conceptualization, drawing attention to the price-efficacy link should make the expectancies in the reduced-price condition parallel to those in the regular-price condition. Indeed, the expectancy ratings were not different across the two price levels $(\mathrm{Ms}=4.3 ; \mathrm{F}<1)$.

Why did we not observe a desirable placebo effect? A question that arises from Experiments 1 and 2 is why there was no desirable placebo effect of the regular-priced drink and an undesirable placebo effect of the reduced-price drink. Part of the answer to this question comes from examining participants' expectancies before the puzzle-solving task in the no-treatment control condition. Note that unlike the treatment participants, the control participants were given neither SoBe nor the accompanying instructions that emphasized the price of the drink before they completed the puzzle-solving task. Thus, beliefs about the price of SoBe could not be activated in these participants. However, as we show in Figure 1, other beliefs (e.g., about participants' natural [unaided] ability in tasks such as solving word puzzles) may have been more salient to control participants. Among treatment participants, these self-efficacy beliefs may have been less salient as a result of the external cues that were presented (e.g., the drink's price having been presented more than once at the beginning of the experiment). In summary, a combination of two reasons may account for our results: (1) Price-related (self-efficacy) beliefs may have been the most salient to participants in the treatment (control) conditions as a result of the procedure we used, and therefore (2) the resulting expectancies related to selfefficacy beliefs in the control conditions may have been as high as those related to price in the regular-price conditions. To test these accounts, we first examined a measure related to self-efficacy beliefs that was collected at the end of the experiment; participants were asked to rate how good they were in general at solving word puzzles on a scale that ranged from 1 ("not at all good") to 7 ("very good"). As we expected, the mean rating on this measure was higher in the control condition $(\mathrm{M}=3.9)$ than in the regular-price condition $(\mathrm{M}=3.2 ; \mathrm{F}(1,189)=4.5, p<.05)$ and the reducedprice condition $(\mathrm{M}=3.3 ; \mathrm{F}(1,189)=3.8, p<.05)$.

To explore this finding further, we conducted a separate study to assess expectancies before the puzzle-solving task (those expectancies were not administered in the control conditions of the main experiments). We had 61 participants from the same population as that in the main studies perform a task that was similar to those of Experiments 1 and 2. Participants were randomly assigned to one of three con- ditions: two treatment conditions (regular price and reduced price) and one no-treatment control condition. After participants received the initial instructions and paid for their drinks (in the treatment conditions), they performed a filler task and then rated their expected concentration and mental performance during the subsequent puzzle-solving task on two scales that we adapted from those administered in the high-expectancy-strength conditions of Experiment 1. As we expected, the mean expectations were not different in the regular-price condition $(\mathrm{M}=4.6)$ and control condition $(\mathrm{M}=4.4 ; \mathrm{F}<1)$ but were lower in the reduced-price condition $(\mathrm{M}=3.3)$ than in the control condition $(\mathrm{F}(1,58)=22.4$, $p<.0001)$. These findings suggest that a possible reason we did not observe a desirable placebo effect of the drink in the regular-price conditions of Experiments 1 and 2 was that the expectations before the puzzle-solving task were not different in these conditions from those in the no-treatment control conditions.

\section{Discussion}

The results of Experiment 2 provide further support for a placebo effect due to price discounts. We replicated the findings of Experiment 1 in the low price-efficacy salience conditions of Experiment 2; that is, the number of puzzles participants solved was lower when they purchased the product at a discounted price than when they purchased it at the regular price. Furthermore, as in Experiment 1, we observed an undesirable placebo effect in the reduced-price condition but not a desirable placebo effect in the regularprice condition, compared with the no-treatment control. Experiment 2 sheds additional light on the underlying process. Specifically, drawing attention to the priceefficacy beliefs before participants solved puzzles reduced the strength of response expectancies, eliminating the undesirable placebo effect we observed in the reduced-price condition. Furthermore, in line with prior work on nonconscious effects of biasing information, when participants' attention was drawn to the relationship between price and the product's efficacy, the placebo effect did not occur. This result supports the notion that the process leading to the placebo phenomenon we observed occurs nonconsciously. Finally, that the basic effect was attenuated rather than enhanced reduces the viability of an alternative account related to cognitive dissonance.

Experiment 2 also sheds light on a potential reason for our observing only an undesirable placebo effect in the first two experiments. First, self-efficacy ratings were higher in the control condition than in the treatment conditions. We discuss this finding further in the "General Discussion" section. Second, a separate study revealed that expectancies in the regular-price condition were not different from those in the no-treatment control, which can account for the finding that the number of puzzles solved was not different across these conditions.

\section{EXPERIMENT 3}

Experiment 3 had several goals. One goal was to obtain further support for the role of response expectancies in the placebo effect of price discounts that we observed. Enhancing the antecedent intrinsic beliefs about the active substance or treatment through advertising claims, for example, should strengthen expectancies and, therefore, the subse- 
quent placebo effect. Accordingly, we manipulated not only the price factor as in the previous two experiments but also another marketing variable related to intrinsic beliefs about the effectiveness of SoBe. We presented the following instructions on the cover page: "Drinks such as SoBe have been shown to improve mental functioning, resulting in improved performance on tasks such as solving puzzles. In fact, the Web site of SoBe includes references to over 50 scientific studies suggesting that consuming drinks like SoBe can significantly improve mental functioning (in the high-expectancy-strength conditions)/slightly improve mental functioning (in the low-expectancy-strength conditions)." We expected that adding this second factor related to expectancy strength would enable us to observe a desirable placebo effect (in the regular-price, high-expectancystrength condition), which we had not observed in the previous two experiments.

A second goal of Experiment 3 was to examine the role of other mediators of the observed effects. Note that in Experiments 1 and 2, we found that response expectancies that were measured before the puzzle-solving task mediated the observed effects. However, we did not examine how respondents felt during the puzzle-solving task. Specifically, did respondents in the regular-price conditions feel more motivated and alert during the task than respondents in the reduced-price conditions? To accomplish the second goal, we administered measures after the puzzle-solving task. We asked participants to rate how alert and how motivated they felt during the puzzle-solving task using seven-point scale items, with the last item reverse scaled.

A third goal of Experiment 3 was to rule out an alternative account related to mood states. It is possible that participants in the reduced-price conditions of Experiments 1 and 2 were in a more positive mood state (because they had received the drink at a discount) than those in the regularprice conditions. Research on mood state effects suggests that positive mood states can impair cognitive capacity and evoke less careful and substantive process styles than less positive mood states (for a review, see Forgas 1995). This might account for why participants in the reduced-price conditions solved fewer puzzles. We tested the validity of this account by collecting measures of mood states (in line with the work of Watson, Clark, and Tellegen [1988]) before participants completed the puzzle-solving task.

To summarize, Experiment 3 used a 2 (price: regular versus discounted) $\times 2$ (expectancy strength: low versus high) between-subjects design and a control condition. Apart from modifying the task to incorporate the expectancystrength factor, including expectancy measures before the puzzle-solving task in all conditions (as in the highexpectancy-strength conditions of Experiment 1) and changing some of the puzzles for logistical reasons, the procedure paralleled those we used in Experiments 1 and 2. Our changes to some of the puzzles may account for some differences in the basic results across Experiments 1, 2, and 3. A total of 204 undergraduate students participated in the study.

\section{Results}

The average number of puzzles solved in the various conditions appears in Figure 4. An ANCOVA on the number of
Figure 4

NUMBER OF PUZZLES SOLVED: EXPERIMENT 3

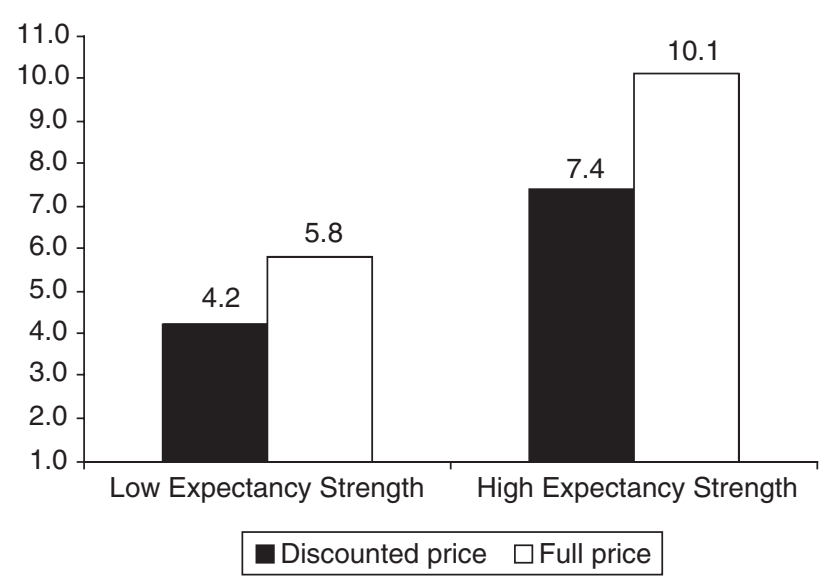

Notes: The number of puzzles solved in the control condition $=6.8$. Before solving the puzzles, participants in all treatment conditions rated their drink-related expectancies as did those in the high-expectancystrength conditions of Experiment 1.

puzzles solved revealed an interaction between price and price-efficacy salience $(\mathrm{F}(1,196)=3.8, p<.05)$ and main effects of price $(\mathrm{F}(1,196)=54.6, p<.0001)$ and expectancy strength $(\mathrm{F}(1,196)=132.8, p<.0001)$. Note that unlike Experiments 1 and 2, prior consumption of $\mathrm{SoBe}$ was not a relevant covariate, probably because virtually all participants indicated that they had consumed this drink before.

The results in the low-expectancy-strength conditions paralleled those in the corresponding conditions of Experiment 1 and the low price-efficacy salience conditions of Experiment 2. As in Experiments 1 and 2 and consistent with a placebo effect, the number of puzzles solved was lower in the reduced-price condition $(\mathrm{M}=4.2)$ than in the regular-price condition $(\mathrm{M}=5.8 ; \mathrm{F}(1,196)=15.1, p<$ $.0001)$ and the control condition $(\mathrm{M}=6.8 ; \mathrm{F}(1,196)=33.1$, $p<.0001)$. In addition, the number of puzzles solved in the regular price, low-expectancy-strength condition was lower $(\mathrm{M}=5.8)$ than in the control condition $(\mathrm{M}=6.8$; $\mathrm{F}(1,196)=3.6, p<.10)$, indicating that presenting weak claims about the efficacy of SoBe resulted in an undesirable placebo effect even when the drink was sold at its regular price.

The results in the high-expectancy-strength conditions were also consistent with our conceptualization. Specifically, presenting strong claims about the efficacy of SoBe to strengthen response expectancies increased the number of puzzles solved in those conditions compared with the low-expectancy-strength conditions. The number of puzzles solved in the reduced-price condition was again lower $(\mathrm{M}=7.4)$ than that in the regular-price condition $(\mathrm{M}=$ $10.1 ; \mathrm{F}(1,196)=42.8, p<.0001)$, but the number was higher in the regular-price condition than in the control condition $(\mathrm{F}(1,196)=73.1, p<.0001)$, a desirable placebo effect that we had not observed in our previous experiments. 
We found further support for our conceptualization by examining the expectancy measures that we collected across all conditions before the puzzle-solving task. In line with our conceptualization, when the expectancy strength was low, these ratings were lower in the reduced-price condition $(\mathrm{M}=3.2)$ than in the regular-price condition $(\mathrm{M}=$ 3.6; $\mathrm{F}(1,196)=3.4, p<.10)$ and the control condition $(\mathrm{M}=$ $4.2 ; \mathrm{F}(1,196)=15.1, p<.0001)$. When the expectancy strength was high, these ratings were again lower in the reduced-price condition $(\mathrm{M}=4.7)$ than in the regular-price condition $(\mathrm{M}=5.9 ; \mathrm{F}(1,196)=25.4, p<.0001)$. Furthermore, a Sobel test revealed that these ratings mediated the effects of the independent variables on the number of puzzles solved $(\mathrm{z}=2.3, p<.05)$.

Alertness and motivation during the task. Recall that after the puzzle-solving task, we asked participants how alert and motivated they felt during the task. Separate Sobel tests revealed that only the alertness measure mediated the effects of the independent variables on the number of puzzles solved $(\mathrm{z}=2.0, p<.05$; in addition, the pattern of results on this measure mirrored that of the number of puzzles solved). This finding is reasonable given that a major claim of this drink is that it boosts alertness. The other measure (which, incidentally, the drink does not claim to influence) was not relevant as a mediator $(p>.60)$.

Did mood mediate the observed effects? An alternative account for our findings is that participants who bought the drink at a discount were in a more positive mood state than those who purchased it at the regular price. However, the mood explanation predicts only a main effect of price and not an interaction with a second factor, such as expectancy strength, that we find in all three experiments. To reduce the viability of the mood account further, we collected measures of mood states before the puzzle-solving task. Separate ANCOVAs with the positive and negative mood measures revealed no relevant effects.

\section{Discussion}

Experiment 3 documented not only an undesirable placebo effect as in our previous experiments but also a desirable placebo effect. Specifically, participants who purchased the drink at its regular price and were presented with strong advertising claims about the drink solved more puzzles than did participants in the control condition. Results of this experiment also suggest that participants felt more alert in the regular-price condition than in the reduced-price conditions and that this mediated the placebo effect, consistent with a claimed effect of the drink (i.e., helping people feel more alert). Finally, Experiment 3 reduces the viability of an alternative account related to mood states. First, as in the previous two experiments, it is difficult to account for the interactive pattern of results that we obtained in this experiment with the mood explanation. Furthermore, the measures of mood states we obtained before the puzzle solving task revealed that participants' mood was not different across the various conditions.

This study suggests the possibility that placebo effects of marketing actions could create ethical dilemmas. For example, a marketer may falsely claim that a product offers a particular benefit, or similarly, a marketer could repackage a product and significantly increase its price, suggesting (explicitly or implicitly) that the higher cost of the cosmeti- cally different product is justified by its greater efficacy. Because of placebo effects of marketing actions, consumers' misplaced beliefs in such seemingly baseless claims may paradoxically make those claims partly true; indeed, given that unlike false statements, puffery is often considered acceptable, even modest placebo effects may make false claims legitimate.

\section{GENERAL DISCUSSION}

Marketing actions can have powerful perceptual effects (e.g., Allison and Uhl 1964; McClure et al. 2004). For example, cola can taste different when a person knows it is "the real thing" (i.e., a Coke) from the same product when it is mislabeled as a generic brand. More generally, it is widely known that marketers can significantly influence variables such as (perceptions of) consumption experiences and purchase behavior. This article extends the scope of effects that marketing actions are known to be capable of evoking, showing that such actions can also influence the actual efficacy of a marketed product. For example, we found that participants who consumed an energy drink purported to improve mental acuity that they purchased at a discounted price subsequently performed worse on a puzzle-solving task than did equivalent participants who purchased the same drink at its regular price.

We provide evidence that the effect of the documented marketing actions is mediated by expectations. For example, in Experiments 1, 2 and 3, we show that varying the strength of response expectancies affects the magnitude of the effect. Specifically, in Experiment 1, we show that performance (after a participant consumed an energy drink he or she purchased at a discount) was worse when the expectations related to the drink's efficacy were strengthened than when they were not. In Experiment 2, drawing participants' attention to their beliefs about the price-efficacy link weakened their beliefs (some respondents presumably realized that the beliefs may not be applicable to that situation) and, thus, the magnitude of the effect. Experiment 3 revealed that strengthening response expectancies by presenting strong advertising claims enhanced the magnitude of the basic effect. Across the three experiments, we also rule out several alternative accounts, such as those relating to cognitive dissonance, distraction, and mood states. Our findings also suggest that the process by which expectations lead to the observed effects occurs nonconsciously.

The effect we document is akin to placebo effects in medicine. We contribute to the placebo literature by extending the types of features that are known to evoke such effects from those that are inherent to the placebo (e.g., information about the placebo, the substance/treatment it replaces, how a placebo is administered) to price, a feature that is not inherent to the placebo. Given placebo researchers' interest in when placebo effects occur nonconsciously, it is also significant that we found that the process by which expectations lead to the placebo effects occurs nonconsciously.

Our findings extend what is known about the association between price and quality in a significant way, showing that price affects not only perceived quality but also actual quality (i.e., the actual efficacy of the product). Indeed, the effect we found and its dependence on expectancies shed light on a puzzling disparity between two conclusions of the 
large body of research on the relationship between price and quality (see Bettman, John, and Scott 1986; Gerstner 1985; John, Scott, and Bettman 1986). On the one hand, there is vast empirical evidence that consumers often perceive lower-priced products and services to be of lower quality, especially if they have no simple alternative way to assess quality (Rao and Monroe 1989). On the other hand, in general, investigations of the relationship between price and objective indications of quality, such as Consumer Reports ratings, arrive at a different conclusion. For example, Riesz (1979) examines the correspondence between prices and indications of objective quality from Consumer Reports for 679 brands in 40 packaged food product classes over a 15year period. He concludes that the correlation was near zero, and in cases such as frozen foods, it was even negative. More generally, such investigations conclude that the empirical relationship between those two variables is weak at best (Gerstner 1985). Why would consumers perceive the relationship between price and quality to be significant when, in general, it is not so? An explanation that is implied by our research for this discrepancy may be a self-fulfilling nature of consumer expectations. Such expectations may lead lower-priced products to perform worse, regardless of whether their objective indications of quality (research of the type that Consumer Reports examines) are actually worse. In other words, the well-known cliché that "one gets what one pays for," may have more merit than has been believed. Exploring this is a worthwhile direction for further research.

A related direction for further research is to delve deeper into why we observed only undesirable placebo effects related to discounted prices in Experiments 1 and 2. Our findings in Experiment 2 suggest that one reason that could account for our results is that people normally focus on selfefficacy beliefs in tasks such as solving puzzles but partly shift their focus away from their own abilities toward beliefs about external stimuli when they are presented with a performance enhancer such as SoBe. An interesting research question is, Can being offered a performance enhancer lower expectations about or the salience of a person's abilities and, thus, potentially lead to diminished performance (particularly if beliefs about the efficacy of the product are not as strong as the self-efficacy beliefs)?

We believe that another promising direction for further research is to demonstrate additional situations in which marketing actions have placebo effects. For example, replicating our results with price promotions on medical products is another worthwhile direction for further research, and there would be considerable implications for the marketing of such products and for public policy. As a first step in this direction, we conducted a small preliminary study. We asked undergraduate marketing students to maintain diaries of when they caught a cold over the course of a semester and used an over-the-counter medication (i.e., a prescription was not necessary) to treat the symptoms. At the end of the semester, 29 students who had fallen ill during the semester and who had bought national over-thecounter brands indicated how effective the medication they had bought was in treating their symptoms on a scale that ranged from 1 ("not at all") to 7 ("very"). In a separate question, we asked them to indicate whether they had bought the medication at its regular price or at a discounted price. Consistent with a placebo effect of price discounts, the 16 students who had bought their medication at a discounted price rated the effectiveness of the medication to be lower $(\mathrm{M}=3.6)$ than the 13 students who had bought their medication at the regular price $(\mathrm{M}=5.5 ; \mathrm{F}(1,27)=18.8$, $p<.01)$.

More generally, it seems reasonable to speculate that marketing decisions ranging from product features, such as color and texture, to marketing-mix decisions, such as advertising messages and distribution channels, may influence the physical effectiveness of the products to which they are applied. If this is the case, the implications could be immense. As an admittedly speculative possibility, if two consumers purchase the same car but one does so at a substantial discount, the two consumers may drive differently. A possible result could be that the consumer who purchases the car at a discount is more accident-prone. Alternatively, if two consumers purchase the same car but only one is exposed to advertising messages that stress the safety benefits of the car, the possible result could be that the consumer exposed to the advertising drives differently than the other consumer. Further research should carefully examine such possibilities.

Another worthwhile research direction is to identify additional moderators of the effect. Beyond the obvious theoretical importance, this would also be significant from a practical viewpoint because it might help reduce or even eliminate undesirable placebo effects (as we did in Experiment 2) in such cases as selling subsidized medications to consumers. For example, will a delay between consumption of a health-related product and subsequent engagement in a task (see Nowlis, Mandel, and McCabe 2004) diminish the magnitude of the placebo effect we document? An investigation of such questions will help identify boundary conditions, shed more light on the underlying process, and explore the scope of placebo effects on marketing actions.

\section{REFERENCES}

Adaval, Rashmi and Kent B. Monroe (2002), "Automatic Construction and Use of Contextual Information for Product and Price Evaluations," Journal of Consumer Research, 28 (March), 572-88.

Allison, Ralph I. and Kenneth P. Uhl (1964), "Influence of Beer Brand Identification on Taste Perception," Journal of Marketing Research, 1 (August), 36-39.

Bettman, James R., Deborah R. John, and Carol A. Scott (1986), "Covariation Assessment by Consumers," Journal of Consumer Research, 13 (December), 316-26.

Bienenfeld, Laura A., William H. Frishman, and Stephen P. Glasser (1996), "The Placebo Effect in Cardiovascular Disease," American Heart Journal, 132 (December), 1207-1221.

Fillmore, Mark and Muriel Vogel-Sprott (1992), "Expected Effect of Caffeine on Motor Performance Predicts the Type of Response to Placebo," Psychopharmacology, 106 (February), 209-214.

Fitzsimons, Gavan J., Tanya L. Chartrand, and Gráinne M. Fitzsimons (2004), "Behavior Responses to Subliminal Brand Exposure," working paper, Fuqua School of Business, Duke University.

Forgas, Joseph P. (1995), "Mood and Judgment: The Affect Infusion Model (AIM)," Psychological Bulletin, 117 (January), 39-66.

Gerstner, Eitan (1985), "Do Higher Prices Signal Higher Quality?" Journal of Marketing Research, 22 (May), 209-215. 
Hahn, Robert A. (1997), "The Nocebo Phenomenon: Scope and Foundations," in Placebo Effect: An Interdisciplinary Exploration, Anne Harrington, ed. Cambridge, MA: Harvard University Press, 56-76.

Huber, Joel and John McCann (1982), "The Impact of Inferential Beliefs on Product Evaluations," Journal of Marketing Research, 19 (August), 324-33.

John, Deborah R., Carol A. Scott, and James R. Bettman (1986), "Sampling Data for Covariation Assessment: The Effect of Prior Beliefs on Search Patterns," Journal of Consumer Research, 13 (June), 38-47.

Kaptchuk, Ted J., Peter Goldman, David A. Stone, and William B. Stason (2000), "Do Medical Devices Have Enhanced Placebo Effects," Journal of Clinical Epidemiology, 53 (August), 786-92.

Kirsch, Irving (1985), "Response Expectancy as a Determinant of Experience and Behavior," American Psychologist, 40 (November), 1189-1202.

(1999), How Expectancies Shape Experience. Washington, DC: American Psychological Association.

- (2004), "Conditioning, Expectancy, and the Placebo Effect: Comment on Stewart-Williams and Podd," Psychological Bulletin, 130 (March), 341-44.

- and Steven Jay Lynn (1999), "Automaticity in Clinical Psychology," American Psychologist, 54 (July), 504-515. and Guy Sapirstein (1999), "Listening to Prozac But Hearing Placebo: A Meta-Analysis of Antidepressant Medications," in How Expectancies Shape Experience, Irving Kirsch, ed. Washington, DC: American Psychological Association, 303-320.

and Lynne J. Weixel (1988), "Double-Blind Versus Deceptive Administration of a Placebo," Behavioral Neuroscience, 102 (April), 319-23.

Levin, Irwin P. and Gary J. Gaeth (1988), "How Consumers Are Affected by the Framing of Attribute Information Before and After Consuming the Product," Journal of Consumer Research, 15 (December), 374-78.

MacKinnon, David P., Chondra M. Lockwood, Jeanne M. Hoffman, Stephen G. West, and Virgil Sheets (2002), "A Comparison of Methods to Test Mediation and Other Intervening Variable Effects," Psychological Methods, 7 (March), 83-104.
McClure, Samuel M., Jiam Li, Damon Tomlin, Kim S. Cypert, Latané M. Montague, and P. Read Montague (2004), "Neural Correlates of Behavioral Preference for Culturally Familiar Drinks," Neuron, 44 (October), 379-87.

Montgomery, Guy and Irving Kirsch (1996), "Mechanisms of Placebo Pain Reduction: An Empirical Investigation," Psychological Science, 7 (May), 174-76.

Nowlis, Stephen M., Naomi Mandel, and Deborah B. McCabe (2004), "The Effect of a Delay Between Choice and Consumption on Consumption Enjoyment," Journal of Consumer Research, 31 (December), 502-510.

Rao, Akshay R. and Kent B. Monroe (1988), "The Moderating Effect of Prior Knowledge on Cue Utilization in Product Evaluations," Journal of Consumer Research, 15 (September), 253-64.

and - (1989), "The Effect of Price, Brand Name, and Store Name on Buyers' Perceptions of Product Quality: An Integrative Review," Journal of Marketing Research, 26 (August), 351-57.

Rescorla, Robert A. (1988), "Pavlovian Conditioning: It's Not What You Think It Is," American Psychologist, 43 (March), 151-60.

Riesz, Peter C. (1979), "Price-Quality Correlations for Packaged Food Products," Journal of Consumer Affairs, 13 (Winter), 236-47.

Rogers, James T. (1985), Dictionary of Clichés. New York: Facts on File Publications.

Stewart-Williams Steve and John Podd (2004), "The Placebo Effect: Dissolving the Expectancy Versus Conditioning Debate," Psychological Bulletin, 130 (March), 324-40.

Strack, Fritz, Norbert Schwarz, Herbert Bless, Almut Kübler, and Michaela Wänke (1993), "Awareness of the Influence as a Determinant of Assimilation Versus Contrast," European Journal of Social Psychology, 23 (January-February), 53-62.

Wager Tor D., James K. Rilling, Edward E. Smith, Alex Sokolik, Kenneth L. Casey, Richard J. Davidson, Stephen M. Kosslyn, Robert M. Rose, and Jonathan D. Cohen (2004), "PlaceboInduced Changes in fMRI in the Anticipation and Experience of Pain," Science, 303 (February), 1162-67.

Watson, David, Lee Anna Clark, and Auke Tellegen (1988), "Development and Validation of Brief Measures of Positive and Negative Affect: The PANAS Scales," Journal of Personality and Social Psychology, 54 (June), 1063-1070. 